\title{
Étude préliminaire sur l'efficacité de différents moyens de lutte contre le piétin-échaudage du gazon (Gaeumannomyces graminis var avenae)
}

\author{
P Lucas, A Sarniguet, N Cavelier*, S Lelarge \\ INRA centre de recherche de Rennes, station de pathologie végétale; "SRIV, BP 29, 35650 Le Rheu, France
}

(Reçu le 5 octobre 1991, accepté le 10 janvier 1992)

\begin{abstract}
Résumé - En France, depuis 1988, de fréquentes et importantes attaques de piétin-échaudage des graminées ont été signalées sur terrains de golf récemment construits. Dans le but de proposer des méthodes de lutte, des expériences ont été menées au laboratoire et en serre. Trois fongicides, 3 souches de Pseudomonas fluorescents et 2 formes d'engrais azoté sont comparés dans la lutte contre le piétin-échaudage sur 2 variétés d'Agrostis palustris (cv Penneagle et Penncross). Les plantes sont cultivées en pots sur du sable infesté avec Gaeumannomyces graminis var avenae. Deux semaines après traitement $(T+2)$, une réduction significative de la maladie est obtenue avec les 3 bactéries (27-45\%), mais non avec les fongicides qui présentent néanmoins une bonne efficacité in vitro. À $T+5$, l'indice de maladie est réduit de $61 \%$ par le triadiménol, $52 \%$ par le nuarimol et $48 \%$ par le cyproconazole, alors qu'une seule souche bactérienne donne de bons résultats à ce stade (réduction de $22 \%$ ). À $T+16$, seuls les fongicides, conservent une efficacité. Aucun effet des apports d'azote nitrique ou ammoniacal n'est observé. Penncross se révèle beaucoup plus sensible au piétin-échaudage que Penneagle. Les résultats médiocres obtenus avec les bactéries et avec les apports d'azote sous différentes formes sont discutés par rapport à la nature du support de culture d'agrostide.
\end{abstract}

gazon / piétin-échaudage / lutte chimique / lutte biologique

\begin{abstract}
Summary - Preliminary studies on the efficacy of some treatments to control take-all patch on turfgrass. Since 1988, take-all patch of turfgrass caused by Gaeumannomyces graminis var, avenae (Gga) has often been reported on recently-constructed golf courses in France. in an attempt to propose methods of control to greenkeepers, experiments were conducted in the laboratory and in the greenhouse. Three fungicides, 3 isolates of fluorescent Pseudomonas, and 2 sources of nitrogen fertilizer were compared on 2 cultivars of Agrostis palustris (Penneagle and Penncross). Plants were grown in pots of sand inoculated with Gga. Two weeks after treatment $(T+2)$, a significant reduction in disease index occurred for the 3 bacteria tested (27-45\%) but not with the fungicides, although they showed a good in vitro activity. At $T+5$, the disease index was reduced by triadimenol (61\%), nuarimol (52\%) and cyproconazole (48\%). At this stage, only 1 bacterium still gave a good control (22\%). At $T+16$, control was obtained only from the fungicides. No effect was observed with either nitrate or ammonium forms of nitrogen. Penncross appeared to be much more susceptible to take-all than Penneagle. The poor results obtained with bacteria may be due to the low nutrient concentration of the sand used as a crop substrate, unable to sustain the population of introduced antagonistic bacteria. The lack of efficacy of the ammonium source of nitrogen may be due to the microbial population of this same substrate being too low to achieve, a sufficient microbiological barrier with ammonium sulphate, against the spread of the disease.
\end{abstract}




\section{INTRODUCTION}

Depuis 1988, sur les terrains de golf, de fréquentes et importantes attaques de piétinéchaudage des graminées, dues à Gaeumannomyces graminis von Arx et Olivier var avenae Turner (Dennis) (Gga), ont été signalées en France (Lucas et al, 1992). Les premiers dégâts observés à Bénodet (Finistère) concernaient aussi bien les "fairways", ensemencés en un mélange de fétuque rouge (Festuca rubra subsp rubra L cv Ludivine $50 \%$, cv Pernille $20 \%$ ) et d'agrostide (Agrostis tenuis Sibth, $30 \%$ ), que les "greens", ensemencés en agrostide (Agrostis palustris Huds cv Penncross). Les plantes sont détruites sur des foyers circulaires, voire semi-circulaires, dont le diamètre peut atteindre plusieurs dizaines de $\mathrm{cm}$. Cette maladie a été décrite dans de nombreux pays (Nilsson et Drew Smith, 1981) et la méthode de lutte la plus souvent préconisée est l'application d'engrais azoté sous forme ammoniacale (sulfate ou phosphate d'ammoniaque) (Davidson et Goss, 1972).

Cette efficacité de la forme ammoniacale d'azote est aussi démontrée dans le cas du piétin-échaudage du blé dû à une autre variété du même champignon, $G$ graminis var tritici Walker (Huber et al, 1968; Christensen et Brett, 1985; Lucas et Collet, 1988). Plus étudié que l'affection sur gazon, le piétinéchaudage du blé a fait l'objet de nombreuses recherches de méthodes de lutte. Ainsi, l'utilisation possible de traitements fongicides des semences a été évaluée (Bockus, 1983; Cavelier et Lucas, 1985; Bateman, 1986). Ces traitements montrent une efficacité partielle et limitée dans le temps. Des tentatives de lutte biologique par utilisation d'une souche hypoagressive du pathogène (Lemaire et al, 1976), ou de bactéries du genre Pseudomonas (Weller et Cook, 1983) ont été également expérimentées, avec des résultats inconstants (Lucas, 1984; Cook, 1985).
Une étude récente des structures de populations de bactéries dans un foyer de piétinéchaudage sur gazon montre d'une part, qu'au centre du foyer où l'on observe une rémission de la maladie, les peuplements en Pseudomonas fluorescents sont plus élevés et, d'autre part, la proportion de bactéries antagonistes in vitro de Gga, à l'intérieur de ces peuplements, est plus grande que dans n'importe quelle autre partie interne (malade) ou externe (saine) du foyer (Sarniguet, 1990; Lucas et al, 1991).

Dans un essai en pots, conduit sous serre, nous nous proposons d'évaluer, dans le cas du piétin-échaudage sur gazon, l'efficacité de ces différents facteurs rapportés dans la littérature: source azotée de fertilisation, traitements fongicides, apport de bactéries antagonistes. Les résultats obtenus et leur valeur pratique seront discutés dans le contexte particulier (économique et technique) des cultures de gazon de parcours de golf.

\section{MATÉRIELS ET MÉTHODES}

\section{Expérimentation in vitro}

Les matières antifongiques expérimentées (tableau I), utilisées sous forme de spécialités commerciales, sont mises en suspension dans de l'eau stérile désionisée, puis incorporées dans un milieu nutritif gélosé (malt $20 \mathrm{~g}$, agar $20 \mathrm{~g}$, eau $1000 \mathrm{ml}$ ) maintenu à $50^{\circ} \mathrm{C}$. Les concentrations finales en chacun des produits sont en progression géométrique de raison 2 , les valeurs extrêmes étant 0,05 et $3,2 \mu \mathrm{g} / \mathrm{ml}$. Ces mélanges sont homogénéisés, puis distribués dans des boîtes de Petri de $10 \mathrm{~cm}$ de diamètre. Après refroidissement, des disques de mycélium de $4 \mathrm{~mm}$ de diamètre sont disposés au centre des boîtes. Pour chaque modalité, 3 répétitions sont réalisées. Les cultures sont placées en incubation à $20^{\circ} \mathrm{C}$ et les diamètres des plages mycéliennes sont mesurés quotidiennement pendant une semaine. À partir de ces

Tableau I. Matières actives, spécialités commerciales, concentrations en matière active et $\mathrm{Cl}_{50}$ des fongicides expérimentés pour lutter contre Gaeumannomyces graminis var avenae.

\begin{tabular}{lccc}
\hline $\begin{array}{c}\text { Matière } \\
\text { active }\end{array}$ & $\begin{array}{c}\text { Spécialité } \\
\text { commerciale }\end{array}$ & $\begin{array}{c}\text { Concentration en } \\
\text { matière active }(\mathrm{g} / \mathrm{l})\end{array}$ & $\begin{array}{c}C / 50 \\
(\mu \mathrm{g} / \mathrm{ml})\end{array}$ \\
\hline Nuarimol & Trimidal & 120 & 0,16 \\
Triadiménol & Bayfidan & 250 & 0,15 \\
Cyproconazole & Alto & 100 & 0,04 \\
\hline
\end{tabular}


notations, une vitesse de croissance journalière est calculée pour chaque modalité. Les concentrations en matière active qui inhibent la vitesse de croissance mycélienne du champignon de $50 \%$ $\left(\mathrm{Cl}_{50}\right)$ sont alors estimées pour chaque fongicide.

\section{Mise en place des cultures}

Cent quatre vingt pots d'une contenance de $500 \mathrm{ml}$ sont remplis de sable de rivière avant d'être ensemencés en Agrostis palustris cv Penncross pour la moitié d'entre eux, cv Penneagle pour l'autre moitié. Les doses utilisées pour le semis sont équivalentes à $2 \mathrm{~g}$ de semences par $\mathrm{m}^{2}$. L'ensemble des pots est ensuite placé sous serre à une température comprise entre 12 et $18^{\circ} \mathrm{C}$. Trois semaines après semis, les cultures sont fertilisées par apport à la surface de chaque pot de $10 \mathrm{ml}$ d'une solution $\mathrm{A}$ : $\left[\mathrm{KH}_{2} \mathrm{PO}_{4}: 1 \mathrm{~g} ; \mathrm{SO}_{4} \mathrm{Mg}, 7 \mathrm{H}_{2} \mathrm{O}\right.$ : 2,16 g; $\mathrm{NaCl}: 117 \mathrm{mg} / \mathrm{l}]$ et $10 \mathrm{ml}$ d'une solution $\mathrm{B}$ : [( $\left.\mathrm{NH}_{4}\right)_{6} \mathrm{Mo}_{7} \mathrm{O}_{24}, 4 \mathrm{H}_{2} \mathrm{O}: 0,5 \mathrm{mg} ; \mathrm{H}_{3} \mathrm{BO}_{4}: 15 \mathrm{mg}$; $\mathrm{MnSO}_{4}, 4 \mathrm{H}_{2} \mathrm{O}: 20 \mathrm{mg} ; \mathrm{CuSO}_{4}, 5 \mathrm{H}_{2} \mathrm{O}: 2,5 \mathrm{mg}$; $\left.\mathrm{ZnSO}_{4}, 7 \mathrm{H}_{2} \mathrm{O}: 100 \mathrm{mg} / \mathrm{l}\right]$.

\section{Inoculation}

L'inoculum utilisé est produit sur grains d'orge préalablement humidifiés par un volume égal d'eau et stérilisés par 2 passages à l'autoclave $\left(115^{\circ} \mathrm{C}\right.$ pendant $1 \mathrm{~h}$ ), à $24 \mathrm{~h}$ d'intervalle. Ce milieu est ensuite ensemencé avec un explantat de Gga récemment isolé. Après 4 semaines d'incubation à $22^{\circ} \mathrm{C}$, l'ensemble est séché à l'air ambiant avant d'être broyé puis tamisé pour ne conserver que les particules comprises entre 1 et $1,5 \mathrm{~mm}$, appelées propagules. L'inoculation des 2 cultures d'agrostide est réalisée avec ces propagules, à raison de 3 propagules par pot pour un tiers d'entre eux, 6 propagules par pot pour un autre tiers. Le tiers restant constitue les témoins non infestés. Ces propagules sont introduites dans les 10 premiers $\mathrm{mm}$ du sol, 4 semaines après le semis, lorsque le couvert végétal est homogène dans tous les pots.

\section{Traitements}

Dix traitements différents répétés 3 fois pour chaque niveau d'inoculation sont comparés. Ils font appel à 3 techniques particulières: choix de la forme azotée de fertilisation, application de fongicides, application de bactéries. Les traitements sont réalisés 4 semaines après inoculation, ce qui correspond à l'apparition des tout premiers symptômes de jaunissement sur feuilles.

\section{Choix de la forme d'azote}

L'incidence de la fertilisation azotée est appréciée en comparant principalement 2 formes, l'une ammoniacale, $\left(\mathrm{NH}_{4}\right)_{2} \mathrm{SO}_{4}$, l'autre nitrique, $\mathrm{Ca}\left(\mathrm{NO}_{3}\right)_{2}$. Deux applications, fournissant chacune $5 \mathrm{mg} \mathrm{N} / \mathrm{pot}$ pour chacun des engrais, sont réalisées respectivement 2 et 8 semaines après semis (soit 2 semaines avant et 4 semaines après inoculation). Les cultures en pots subissant les autres traitements (bactéries, fongicides) reçoivent la même quantité d'azote sous une forme mixte, ammonitrique $\left[\mathrm{NH}_{4} \mathrm{NO}_{3}\right]$, aux mêmes dates.

\section{Application de fongicides}

Trois fongicides (tableau I) sont appliqués, par pulvérisations des cultures, 8 semaines après semis, soit 4 semaines après l'inoculation avec Gga, aux doses suivantes de matière active: triadiménol $0,2 \mathrm{~g} / \mathrm{m}^{2}$, nuarimol $0,1 \mathrm{~g} / \mathrm{m}^{2}$, cyproconazole $0,025 \mathrm{~g} / \mathrm{m}^{2}$.

\section{Application de bactéries}

L'incidence sur la maladie de l'application de 3 souches bactériennes appartenant au groupe des Pseudomonas fluorescents et isolées d'un échantillon de sol prélevé dans un foyer de piétin-échaudage (Sarniguet, 1990) est appréciée. Deux de ces souches bactériennes (Ben01, Ben02) présentent in vitro une activité antagoniste vis-à-vis de Gga, la troisième (Ben03) n'affecte pas la croissance in vitro de Gga (résultats non présentés). Les bactéries sont multipliées sur milieu LPGA (levure $5 \mathrm{~g}$, peptone $5 \mathrm{~g}$, glucose $5 \mathrm{~g}$, agar $15 \mathrm{~g}$, eau $1000 \mathrm{ml}$ ) pendant $24 \mathrm{~h}$ et mises en suspension dans l'eau stérile. Le titre de chaque suspension de bactéries est ajusté de façon à apporter, à chaque application, $10^{7}$ bactéries $/ g$ de sol en versant $30 \mathrm{ml}$ de cette suspension à la surface de la culture. Deux applications sont réalisées respectivement 8 et 9 semaines après semis, soit 4 et 5 semaines après inoculation.

\section{Dispositif expérimental et notations}

Le dispositif adopté est un dispositif factoriel (3 facteurs: quantités d'inoculum, cultivars, traitements) avec 3 répétitions et randomisation totale. Trois notations ont été réalisées 6,9 et 20 semaines après inoculation soit 2,5 et 16 semaines après traitements fongicides. Une note est attribuée selon l'état de la culture pour chaque traite- 
ment et chaque répétition d'après l'échelle suivante: 0 : culture saine et $1,2,3,4,5$ pour respectivement 1 à 10,11 à 25,26 à 50,51 à 75 , 76 à $100 \%$ de la culture détruite. Les résultats sont soumis à une analyse de variance et les moyennes comparées par le test de Newmann-Keuls.

\section{RÉSULTATS}

\section{Efficacité in vitro des fongicides vis-à-vis de Gga}

Le cyproconazole (tableau I) apparaît comme le produit le plus efficace in vitro. Les 2 autres fongicides ont des $\mathrm{Cl}_{50} 4-5$ fois plus élevées mais toutefois très faibles, justifiant leur expérimentation in situ.

\section{Efficacité des différents traitements in situ}

Les cultures non inoculées en Gga n'ont développé aucun symptôme de maladie, quel que soit le traitement réalisé. Pour les cultures inoculées, les niveaux de maladie ont différé légèrement en fonction de la quantité d'inoculum introduit ( 3 ou 6 propagules) (tableau II) sans interagir avec les effets des traitements ou des cultivars. Les résultats obtenus lors des 3 notations et concernant les facteurs "cultivars » et " traitements", regroupés dans le tableau II, sont donc les valeurs moyennes d'indices de maladie pour les 2 niveaux d'inoculum. La figure 1 illustre les interactions « cultivar $x$ traitement ».

Bien qu'en fin d'expérimentation, à $(T+16)$, leurs indices de maladie soient similaires, le cultivar Penncross est plus sensible que le cultivar Penneagle (tableau II). Les 3 souches bactériennes limitent l'extension des premiers symptômes de piétin-échaudage (notation 2 semaines après traitements). Par contre, à la deuxième notation, 3 semaines plus tard, cet effet n'est observable que sur les cultures traitées avec la bactérie BenO2. À l'inverse, si à la première notation aucune activité des fongicides n'est détectable, à la deuxième, les 3 fongicides montrent une efficacité significative en réduisant l'importance de la maladie. Cette efficacité est encore très nette, 16 semaines après traitement, alors que les différences entre cultivars s'estompent.

Les applications d'azote uniquement ammoniacal, ou uniquement nitrique n'ont, par contre, aucun effet comparées à l'apport d'ammonitrate.

Tableau II. Effet des différents niveaux de facteurs expérimentés (tous autres facteurs confondus), sur le développement du piétin-échaudage sur agrostide, notés 2,5 et 16 semaines après la réalisation des traitements fongicides $(T+2, T+5, T+16)$. Pour chaque facteur analysé, les valeurs suivies de lettres identiques ne sont pas significatives pour $p=0,05$. Échelle de notation: 0 (culture saine) -5 (culture détruite).

\begin{tabular}{lllll}
\hline \multirow{2}{*}{ Facteurs } & \multicolumn{1}{c}{ Modalités } & \multicolumn{3}{c}{ Date de notation } \\
\cline { 3 - 5 } & & $T+2$ & $T+5$ & $T+16$ \\
\hline Inoculations & 6 propagules & $2,09^{\mathrm{a}}$ & $2,00^{\mathrm{a}}$ & $3,19^{\mathrm{a}}$ \\
Gga & 3 propagules & $1,84^{\mathrm{b}}$ & $1,81^{\mathrm{b}}$ & $2,70^{\mathrm{b}}$ \\
Cultivars & Penncross & $2,68^{\mathrm{a}}$ & $2,42^{\mathrm{a}}$ & $2,72^{\mathrm{a}}$ \\
& Penneagle & $1,25^{\mathrm{b}}$ & $1,30^{\mathrm{b}}$ & $2,80^{\mathrm{a}}$ \\
Traitements & Témoin & $2,21^{\mathrm{ab}}$ & $2,35^{\mathrm{a}}$ & $4,25^{\mathrm{ab}}$ \\
& Ca(NO3)2 & $2,65^{\mathrm{a}}$ & $2,65^{\mathrm{a}}$ & $3,17^{\mathrm{ab}}$ \\
& (NH4)2SO $_{4}$ & $2,29^{\mathrm{ab}}$ & $2,47^{\mathrm{a}}$ & $3,83^{\mathrm{b}}$ \\
& Nuarimol & $2,24^{\mathrm{ab}}$ & $1,13^{\mathrm{cd}}$ & $1,23^{\mathrm{d}}$ \\
& Triadiménol & $2,03^{\mathrm{bc}}$ & $0,89^{\mathrm{d}}$ & $0,75^{\mathrm{cd}}$ \\
& Cyproconazole & $2,01^{\mathrm{bc}}$ & $1,22^{\mathrm{cd}}$ & $4,75^{\mathrm{a}}$ \\
& Ben01 & $1,39^{\mathrm{d}}$ & $2,28^{\mathrm{a}}$ & $4,08^{\mathrm{ab}}$ \\
& Ben02 & $1,22^{\mathrm{d}}$ & $1,76^{\mathrm{b}}$ & $3,67^{\mathrm{b}}$ \\
\hline
\end{tabular}



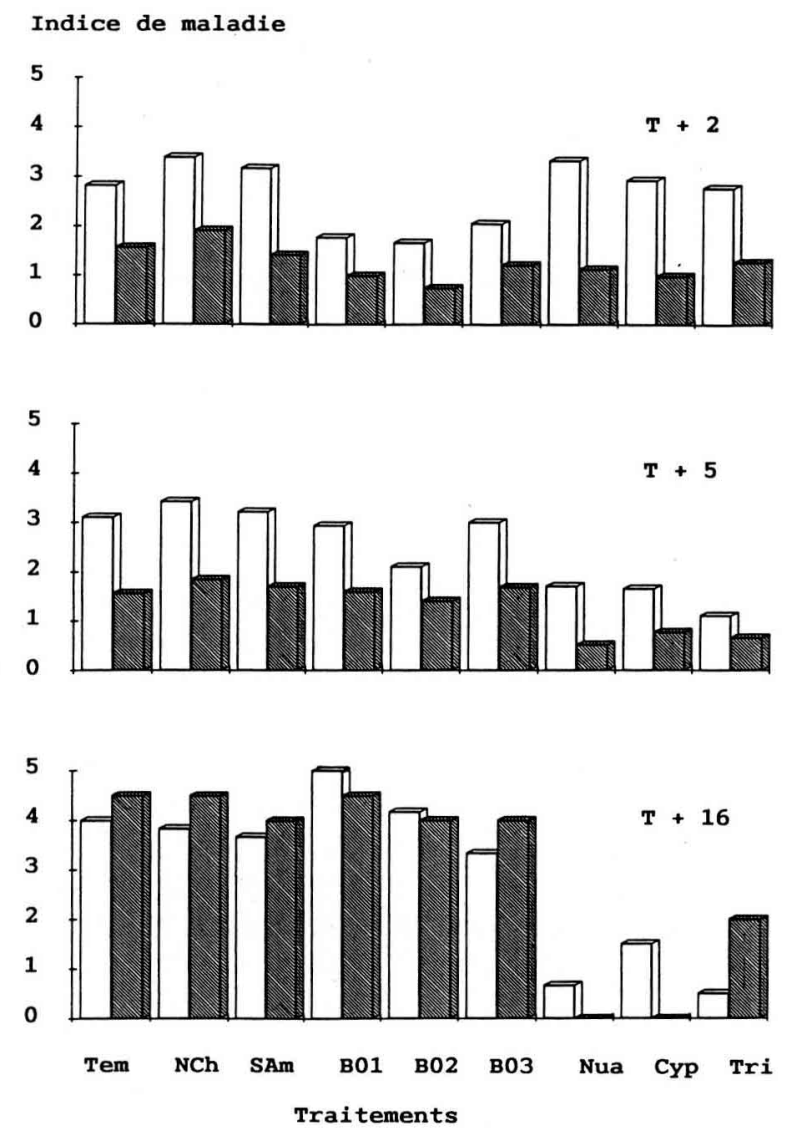

Fig 1. Indices de piétin-échaudage obtenus sur 2 cultivars d'agrostide cultivés en serre et recevant différents traitements $(\mathrm{Tem}=$ Témoin, $\mathrm{NCh}=$ Nitrate de chaux, SAm = Sulfate d'ammoniaque, B01 = bactérie Ben01, $\mathrm{B} 02$ = bactérie $\mathrm{Ben02}, \mathrm{B} 03$ = bactérie Ben03, Nua = Nuarimol, Cyp $=$ Cyproconazole, Tri $=$ Triadiménol). Les notations sont réalisées 2,5 et 16 semaines après la date d'application des traitements fongicides $(T+2, T+5$ et $T+16)$. Agrostis palustris var Penncross $\square$; var Penneagle

\section{DISCUSSION ET CONCLUSIONS}

En premier lieu, il est intéressant de constater que le choix du cultivar d'agrostide permet à lui seul de réduire de façon notable le niveau de maladie. Le cultivar Penneagle apparaît beaucoup moins sensible à la maladie que le cultivar Penncross habituellement cultivé sur les « greens" de golf ensemencés en agrostide.

La très précoce efficacité obtenue avec les bactéries peut être expliquée par l'introduction, à 2 reprises et à 7 jours d'intervalle, d'une grande quantité de bactéries $\left(2.10^{7}\right.$ CFU/g de sol). La perte d'efficacité observée par la suite $\left(2^{e}\right.$ notation) est probablement due à un faible taux de survie de ces bactéries après leur introduction. En effet, le sable de rivière utilisé comme substrat de culture dans cette étude était vraisemblablement peu susceptible de fournir les ressources organiques nécessaires au maintien d'une importante population bactérienne. La même raison peut être à l'origine de l'inefficacité de l'apport d'azote sous forme ammoniacale, ce qui apparaît contradictoire avec les résultats obtenus sur le golf de Bénodet (non présentés ici) ou rapportés dans la littérature (Davidson et Goss, 1972). En effet, une des hypothèses avancées dans le cas du piétin-échaudage du blé pour expliquer l'action des apports d'azote ammoniacal est la stimulation de fractions microbiennes auxiliaires ayant une activité antagoniste contre le pathogène (Smiley, 1978; Sarniguet, 1990). Il est possible que le sable de rivière, supportant une culture pour la première fois, n'ait pas hébergé les populations microbiennes antagonistes nécessaires à l'expression de ce phénomène.

L'effet des fongicides expérimentés est quant à lui plus long à se manifester mais plus durable: 5 semaines après les traitements, la maladie est significativement réduite, quel que soit le fongicide utilisé et cet effet est encore observable 16 semaines après traitements. La meilleure efficacité est obtenue avec le triadiménol, fongicide qui a été utilisé avec succès sur le terrain de golf de Bénodet (résultats non présentés).

Ces résultats ouvrent des perspectives intéressantes pour lutter contre le piétin-échaudage des gazons. À court terme, le choix d'un cultivar moins sensible (Penneagle) s'impose, et il serait intéressant d'apprécier la sensibilité d'autres cultivars d'A palustris au piétinéchaudage. Une protection complémentaire peut être assurée, si nécessaire, par l'application de fongicides et, au vu des premiers résultats obtenus dans cette étude, il paraît possible d'alterner différentes matières actives dans le souci d'éviter l'apparition de phénomènes de résistance, sans perte significative d'efficacité. Enfin, compte tenu des conditions, discutées plus haut, dans lesquelles les résultats avec les bactéries ont été obtenus, ces recherches méritent d'être poursuivies. II serait tout à fait réaliste d'imaginer une méthode de lutte biologique contre cette maladie, les contextes économique et technique d'un parcours de golf étant moins limitants en termes de coût et de nombre d'interventions que ceux d'une culture de blé, par exemple. Par ailleurs, la plupart des " greens " de golf récemment construits sont 
ensemencés sur des substrats de composition relativement proche, donc a priori de réceptivité analogue aux agents de lutte biologique. Enfin la réduction de l'utilisation des pesticides, liée à une telle méthode, est un élément important et valorisant pour une structure de loisir.

\section{RÉFÉRENCES}

Bateman GL (1986) Effects of triadimenol-containing seed treatment on winter wheat infected with take-all. Z Pflanzenkr Pfanzenschutz 93, 404-414

Bockus WW (1983) Effects of fall infection by Gaeumannomyces graminis var tritici and triadimenol seed treatment on severity of take-all in winter wheat. Phytopathology 73, 540-543

Cavelier N, Lucas P (1985) Efficacité du triadiménol sur le piétin-échaudage et sur d'autres maladies du pied des céréales. In: Fungicides for Crop Protection. 100 Years of Progress. Br Crop Protec Council Monograph $n^{0} 31$. BCPC Croydon, 347-350

Christensen NW, Brett M (1985) Chloride and liming effects on soil nitrogen form and take-all on wheat. Agron $J 77,157-163$

Cook RJ (1985) Biological control of plant pathogens with special reference to the take-all fungus in suppressive soils. Plant Prot Bull 27, 179-198

Davidson RM Jr, Goss RL (1972) Effects of P, S, $\mathrm{N}$, lime, chlordane and fungicides on Ophiobo lus patch disease of turf. Plant Dis Rep 56, 565567

Huber DM, Painter CC, McKay HC, Petersen DL (1968) Effect of nitrogen fertilization on take-all of winter wheat. Phytopathology 58, 1470-1478

Lemaire JM, Jouan $B$, Coppenet $M$, Perraton B, Lecorre L (1976) Lutte biologique contre le pié- tin-échaudage des céréales par l'utilisation de souches hypoagressives d'Ophiobolus graminis. Le caractère hypoagressif est-il contagieux? Sci Agron Rennes, 63-66

Lucas $P$ (1984) Lutte contre le piétin-échaudage des céréales par utilisation de souches hypoagressives de Gaeumannomyces graminis var tritici. In: Faune et Flore Auxiliaires en Agriculture. Acta, 171-174

Lucas P, Collet JM (1988) Influence de la fertilisation azotée sur la réceptivité d'un sol au piétin-échaudage, le développement de la maladie au champ et les populations de Pseudomonas fluorescents. Bull OEPP/EPPO Bull 18, 103-109

Lucas $P$, Sarniguet A, Lelarge S (1991) Bacterial populations related to the progress of the disease in take-all patches on turf grass. In: Biotic interactions and soil-borne diseases. (ABR Beemster, GJ Bollen, M Gerlagh, MA Ruissen, B schippers, A Tempel, eds) Elsevier, Amsterdam, 264-270

Lucas P, Sarniguet A, Laurent C (1992) Manifestation en France du piétin-échaudage sur gazon dû à Gaeumannomyces graminis (Sacc) von Arx et Olivier var avenae (Turner) Dennis. Agronomie 12, 183-186

Nilsson HE, Drew Smith J (1981) Take-all of grasses. In: Biology and Control of Take-All. (MJC Asher, PJ Shipton eds) Acad Press, Londres, 433-451

Sarniguet A (1990) Réceptivité des sols au piétinéchaudage du blé: influence des rotations et de la fertilisation azotée en relation avec certains facteurs physicochimiques et les peuplements de Pseudomonas fluorescents. Thèse Doctorat Univ Paris-Sud, 98p

Smiley RW (1978) Antagonists of Gaeumannomyces graminis from the rhizoplan of wheat in soils fertilized with ammonium or nitrate nitrogen. Soil Biol Biochem 10, 169-174

Weller DM, Cook RJ (1983) Suppression of take-all of wheat by seed treatment with fluorescent pseudomonads. Phytopathology 73, 463-469 